\title{
Long-term Transmission Characteristics of CYTOP Fiber Exposed to Gamma Radiation
}

\author{
Ivan Chapalo ${ }^{1}$, Andrei Gusarov ${ }^{2}$, Damien Kinet ${ }^{1}$, Karima Chah $^{1}$, Ying-Gang Nan ${ }^{1}$ and Patrice Mégret ${ }^{1}$ \\ ${ }^{1}$ Electromagnetism and Telecom Department, University of Mons, Belgium \\ ${ }^{2}$ SCK-CEN, Belgium \\ Ivan.Chapalo@umons.ac.be
}

\begin{abstract}
We investigated post-irradiation variations of the radiation induced attenuation (RIA) of polymer perfluorinated (CYTOP) optical fiber exposed to gamma radiation. The RIA measured five months after the irradiation was qualitatively similar to that measured several hours after irradiation. However, quantitatively the RIA post-irradiation evolution significantly depends on the spectral range: in the visible range, the transmission partly recovers, while in the infrared, at wavelengths above $1300 \mathrm{~nm}$ the RIA grows and then saturates becoming permanent. The latter feature is prospective for gamma radiation dosimetry as a permanent long-term indicator of received radiation dose.
\end{abstract}

Keywords - CYTOP, perfluorinated fiber, polymer optical fiber, gamma radiation, radiation induced attenuation, fiber dosimetry.

\section{INTRODUCTION}

$\mathrm{P}$ OLYMER optical fibers (POFs) have been attracting substantial attention from research and industry thanks to particular mechanical properties, simple handling, biomedical compatibility and safety [1,2]. Among POFs, cyclic transparent amorphous fluoropolymer (CYTOP) fiber demonstrates radically low attenuation in the telecom transparency windows of 850 and $1310 \mathrm{~nm}$, thus becoming an efficient solution for short distance communication links [3]. Besides fiber communications, CYTOP fiber has also been intensively studied for various sensing applications: fiber Bragg gratings [4], Brillouin scattering [5] and intermodal interference [6] are good examples of investigated sensing principles.

A particular topic of research is the use of POFs in radiation environments, including possible solutions for gamma radiation dosimetry $[7,8]$. A basic step in this direction is the study of gamma radiation influence on fibers' properties aiming for evaluation of their transmission characteristics degradation. Recently, the gamma-radiation induced attenuation (RIA) in CYTOP fibers has been extensively investigated. The RIA was measured in the visible and near-infrared spectral bands $[9,10]$; the influence of temperature and relative humidity on irradiated fibers was examined [11], and the distributed gamma radiation detector was proposed [7]. However, evolution of the RIA properties versus time was not considered in detail.

In this work, we investigate the long-term (residual) RIA of CYTOP fiber. We irradiated several POF samples by gamma radiation to $1,5,20,50 \mathrm{kGy}$ doses and measured the RIA five months after irradiation in the range of 500-1700 nm. Additionally, we investigate and analyze the RIA timeevolution measured starting shortly after irradiation and during up to 80 hours.

\section{EXPERIMENTAL SETUP}

In experiments, we used multimode graded-index fiber (MMF) GigaPOF-50SR with 50- $\mu \mathrm{m}$ CYTOP core and 490- $\mu \mathrm{m}$ polycarbonate overclad (Chromis Technologies). Several 2-m POF samples were irradiated up to $1 \mathrm{kGy}, 5 \mathrm{kGy}, 20 \mathrm{kGy}$ and $50 \mathrm{kGy}$ gamma radiation doses at a $7.5 \mathrm{kGy} / \mathrm{h}$ dose-rate. Irradiation was conducted using a calibrated setup (Brigitte facility, SCK-CEN, Belgium) based on ${ }^{60} \mathrm{Co}$ irradiation sources located at a depth of seven meters in a water pool (Fig. 1). The sources formed a cylindrical volume where the stainless steel hermetic container with fiber samples was placed for a specified time according to the required dose. After irradiation, the samples were stored at uncontrolled laboratory conditions during five months.

To measure POF transmission characteristics in a wide spectral range, we developed the scheme based on a supercontinuum broadband source (BS) NKT SuperK Compact 450-2400 nm and an optical spectrum analyzer (OSA) Yokogawa AQ6374 350-1750 nm (Fig. 2). The BS was

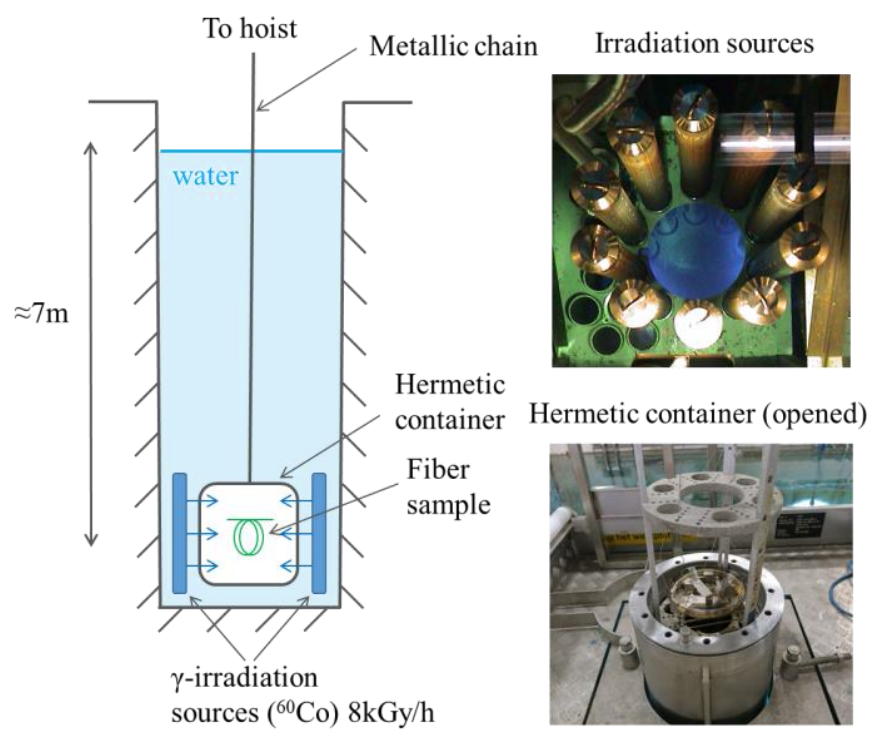

Fig. 1. Schematic and photographs of the irradiation setup. 


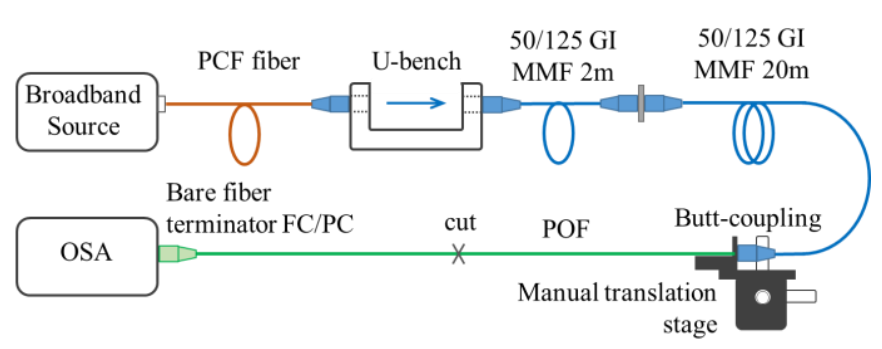

Fig. 2. Schematic of the experimental setup.

equipped with a "U-bench" with collimating optics and FC/APC connectors to avoid mechanical contact and possible damage of delicate PCF-fiber patchcord serving as a BS output. Light was introduced to the POF sample from the FC/PC connector of silica graded-index 50/125 MMF patchcord (GIF50C, Thorlabs) by butt-coupling method at the manual translation stage (NanoMAX 313D, Thorlabs).

The co-axial positioning of the fibers was obtained by observing the speckle pattern of the near-field image at the output POF facet and minimizing the number of interference spots at the speckle pattern. For this, we used visible light source $(635 \mathrm{~nm})$ and silica SMF patchcord (SM600 fiber, Thorlabs) connected to the silica MMF to provide near singlemode launch, when the POF and the MMF were well-aligned. Additionally, fiber positioning was adjusted by maximizing output power from the POF using the OSA in the single wavelength mode. Index-matching gel was utilized at the POF and silica fiber joint. The output end of POF was connected to the OSA using FC/PC bare fiber terminator with a ferrule's internal diameter of $500 \mu \mathrm{m}$.

The losses measurement for each POF sample was conducted by cut-back method: we measured the transmission spectrum at the output of 2-m POF sample, then we cut one meter of the sample and measured the transmission spectrum again. When it was required (for example, in the case of very low signal), the procedure was repeated for $1 \mathrm{~m}$ and so on. Since the output end of the sample was connectorized by the bare fiber terminator, it can bring some measurement inaccuracies. To avoid significant measurement errors, each time we connectorized the POF endface 3-5 times and measured the transmission spectrum for each connectorization. To obtain the final transmission spectrum, we excluded spectra that demonstrated noticeably less power than others and we averaged the remaining spectra.

We cut POFs by a FiberFin razor cutter. After cutting, fiber facets were cleaned by alcohol and inspected with a microscope. The cutting was repeated if visible disturbances of fiber facets were observed.

Multimode nature of CYTOP fiber caused some difficulties with the OSA operation: the spectrum contained parasitic intensity modulation as a result of intermodal interference. The parasitic effect was minimized by two parameters: the fiber length and the OSA resolution. We used additional 20-m 50/125 silica MMF patchcord prior to the POF to increase the fiber length. This made the intermodal interference signal more sensitive to the optical frequency and a modulation in the OSA spectrum became more quickly changing with a wavelength. Then, we choose a resolution of the OSA of $2 \mathrm{~nm}$ so that the parasitic modulation was effectively averaged on the one hand, and spectral peaks inherent in irradiated fibers were not hidden on the other hand. We also supposed that light entering the OSA from the POF end facet was less subjected to spatial filtering by the OSA internal slits when using lower resolution $(2 \mathrm{~nm})$ and therefore the intermodal interference signal was less pronounced.

The RIA was calculated as follows. First, the loss spectrum of irradiated fiber $\alpha_{i r r}$. was calculated from cut-back measurement by subtracting 2-m sample spectrum $S_{1}$ from 1-m sample spectrum $S_{2}$ and dividing by cut length $l(1 \mathrm{~m}$ in this case):

$$
\alpha_{\text {irr. }}[\mathrm{dB} / \mathrm{m}]=\left(S_{2}[\mathrm{dBm}]-S_{1}[\mathrm{dBm}]\right) / l[\mathrm{~m}] .
$$

Then, the RIA spectrum was calculated by subtraction the POF loss spectrum $\alpha_{\text {СYTOP }}$ from the irradiated POF loss spectrum $\alpha_{i r r}$ :

RIA $[\mathrm{dB} / \mathrm{m}]=\alpha_{i r r}[\mathrm{~dB} / \mathrm{m}]-\alpha_{C Y T O P}[\mathrm{~dB} / \mathrm{m}]$.

The POF loss spectrum $\alpha_{C Y T O P}$ was measured in advance by cutback method using 35-m fiber cut to a length of $2 \mathrm{~m}$.

\section{EXPERIMENTAL RESULTS AND DISCUSSION}

Figure 3 presents typical measured raw spectra: the spectrum of the light source (dashed black line), transmission spectrum of 2-m pristine POF sample, and transmission spectra of the POF sample that received a dose of $20 \mathrm{kGy}$ (red and green lines). The irradiated 1-m POF sample was cut to $0.5 \mathrm{~m}$ for the cut-back measurement in this example. It is seen that the light source power spectral density rapidly decreases below $550 \mathrm{~nm}$; irradiated POF demonstrates strong losses in this range as well. Moreover, in the range of $350-550 \mathrm{~nm}$ some oscillating structure independent on fiber length was observed. We interpreted it as a "ghost" spectra according the user's manual of the OSA. Taking the above into account, the lower wavelength of the RIA curves was varied depending on loss level in this spectral region. It also should be mentioned that the light source' power was unstable in a range of 1000-1130 nm, therefore, at the RIA graphs, we did not plot data in this range.

Figure 4 demonstrates RIA graphs measured 5 months after irradiation for doses 1, 5, 20, $50 \mathrm{kGy}$. These measurements were performed at UMons. For convenience of total loss estimation, the pristine CYTOP fiber loss curve is presented as well using additional vertical axis. Three typical zones of RIA spectra can be distinguished: visible and the initial part of infrared with strong RIA increasing towards shorter

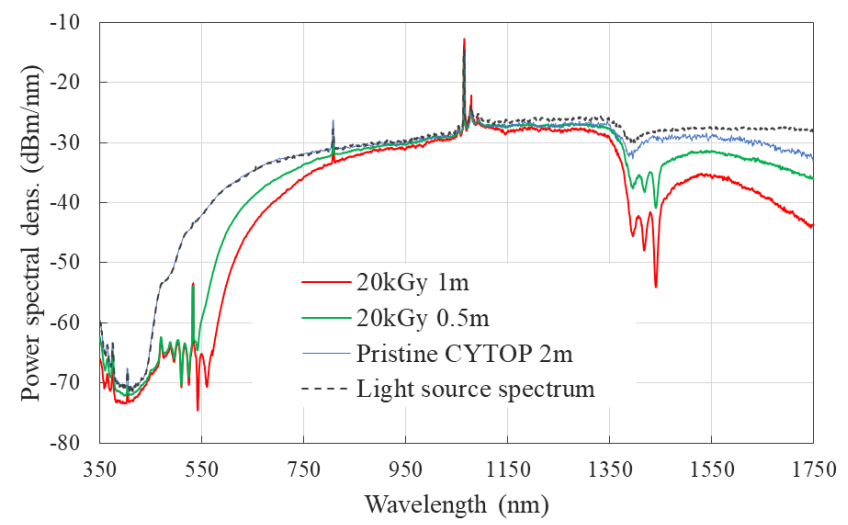

Fig. 3. Typical spectra of the light source, pristine 2-m POF, and irradiated POFs during the cut-back loss measurement. 


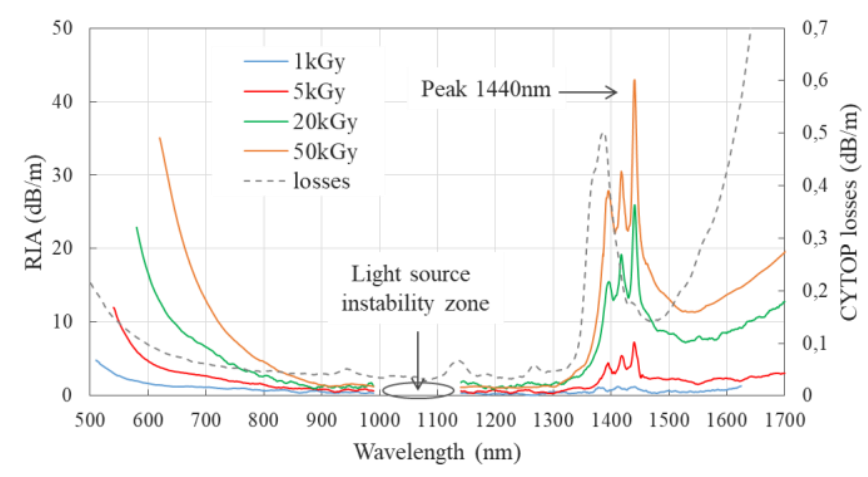

Fig. 4. CYTOP fiber RIA measured 5 months after irradiation.

wavelengths, the zone $900-1300 \mathrm{~nm}$ with a relatively weak RIA, and the zone above $1300 \mathrm{~nm}$ having complex behavior with a few sharp attenuation peaks in the vicinity of $1400 \mathrm{~nm}$. The RIA dependence on the irradiation dose (measured 5 months after irradiation) is presented in Fig. 5 for two representative wavelengths: $650 \mathrm{~nm}$ chosen in the zone of high RIA in the visible part of the RIA spectrum, and the RIA maximum peak of $1440 \mathrm{~nm}$. The dependence at $650 \mathrm{~nm}$ is well approximated by a linear function with a slope $0.46 \mathrm{~dB} /(\mathrm{m} \bullet \mathrm{kGy})$. For the peak wavelength of $1440 \mathrm{~nm}$, the results demonstrate more complex behavior: at the lower doses $(1,5,20 \mathrm{kGy})$ the dependency is close to linear (estimated as $1.27 \mathrm{~dB} /(\mathrm{m} \bullet \mathrm{kGy})$, while at $50 \mathrm{kGy}$ a lower sensitivity was observed. This behavior is not completely clear and should be investigated in more detail with a higher number of irradiation doses. It should be mentioned that at other wavelengths in the visible range, the RIA also demonstrated linear dependency on the irradiation dose but with different sensitivity: the sensitivity increases with decreasing wavelength.

We investigated the influence of launching conditions on the measured RIA. For this, we utilized the superluminescent diode (S5FC1005S, Thorlabs) operating at $1550 \mathrm{~nm}$ with a singlemode output and the mode scrambler (ModCon, Arden Photonics) providing stable multimode regime of light propagation. The RIA measurements using underfilled launch (by the single-mode patchcord) and multimode launch (using the mode scrambler) did not demonstrate noticeable differences in the RIA.

We also investigated the RIA evolution during first hours after irradiation. The irradiation was performed at the same irradiation facility described earlier. To allow for comparison with the previously analyzed long-term RIA, we re-assembled
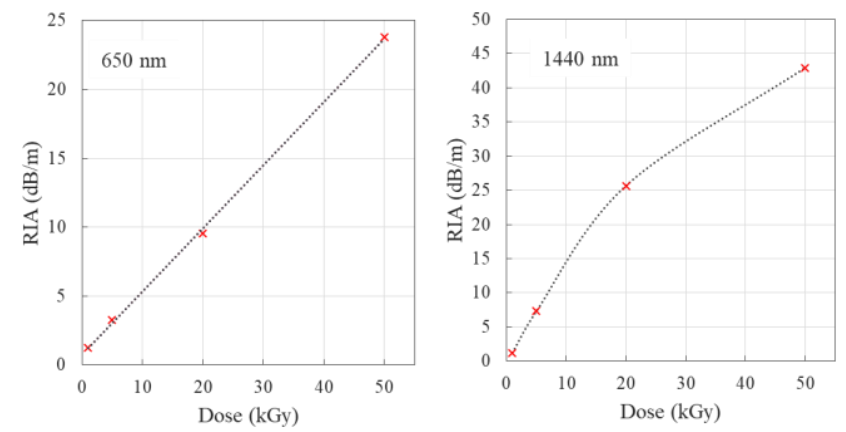

Fig. 5. RIA dependency on the irradiation dose at two wavelengths. the same measurement setup (Fig. 2) at the irradiation site of SCK-CEN. This allowed us to start experiment within 1-2 hours after irradiation. The delay was defined by the safety procedures. We irradiated two 2-m fiber samples up to 5 and $17.5 \mathrm{kGy}$ doses and measured the RIA during more than 20 hours. During the experiment, the temperature was in the range of 27 to $29{ }^{\circ} \mathrm{C}$. The RIA was calculated by subtraction the transmission spectrum of irradiated fiber sample $S_{i r r}$. from the spectrum of the same sample measured before irradiation $S_{\text {ref. }}$ and normalizing by the sample length $l$ :

$\operatorname{RIA}(t)[\mathrm{dB} / \mathrm{m}]=\left(S_{\text {ref. }}[\mathrm{dBm}]-S_{\text {irr. }}(t)[\mathrm{dBm}]\right) / l[\mathrm{~m}]$.

After irradiation and the RIA measurements, the samples were again stored at room conditions during several months in order to measure the long-term RIA.

Measured RIA is presented in Fig. 6. It is seen that after irradiation, significantly stronger RIA (comparing to results obtained 5 months after irradiation) is observed at the shortwavelength part of the spectrum. It, however, drastically decreases with time as a result of annealing process. For example, for $5 \mathrm{kGy}$ dose, the RIA at $850 \mathrm{~nm}$ decreased from $10 \mathrm{~dB} / \mathrm{m}$ measured 1.5 hours after irradiation to $2.5 \mathrm{~dB} / \mathrm{m}$ measured 80 hours after irradiation. Moreover, 5 months after irradiation, the RIA was stabilized at the value less than $1 \mathrm{~dB} / \mathrm{m}$.

In contrast, opposite behavior was observed at the longwavelength part: the RIA continued to rise after irradiation and saturated at levels significantly higher than initial ones in both $1400 \mathrm{~nm}$ sharp peaks region and wavelengths longer than these peaks. This is especially pronounced for the higher dose of $17.5 \mathrm{kGy}$ (Fig. 6b). Unlike the effect of the transmission recovery with time in the visible spectral range, which is typical for various types of fibers and for CYTOP in particular, the
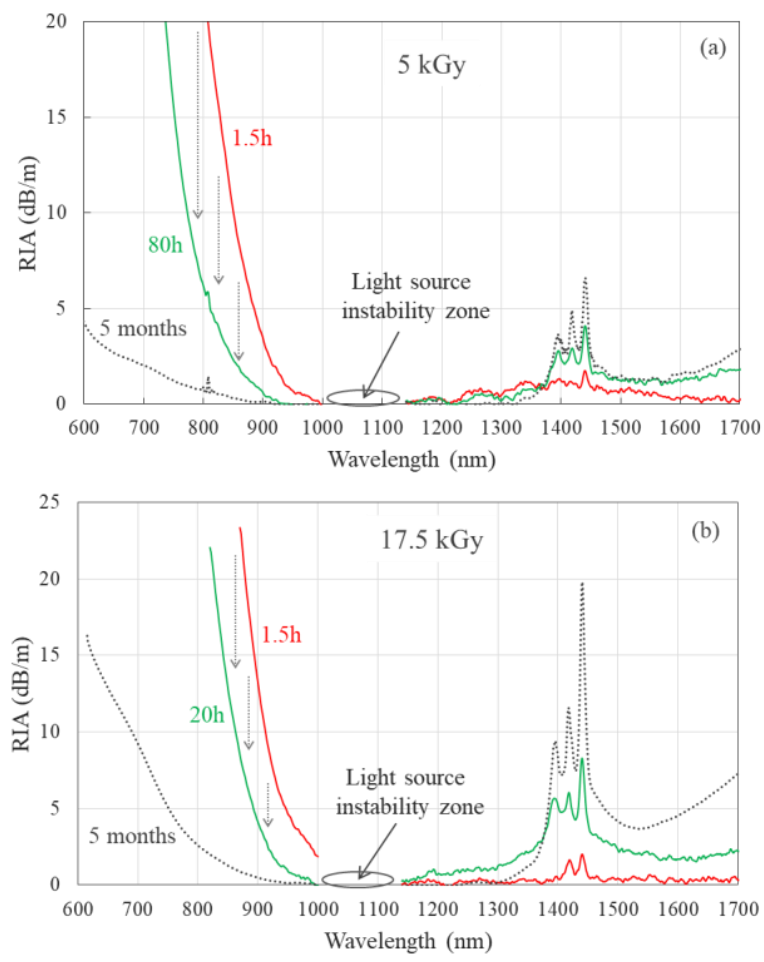

Fig. 6. RIA measured $1.5 \mathrm{~h}, 80 \mathrm{~h}$ and 5 months after irradiation for $5 \mathrm{kGy}$ dose (a) and $1.5 \mathrm{~h}, 20 \mathrm{~h}$ and 5 months after irradiation for 17.5 kGy dose (b). 
continued rise of attenuation after irradiation is quite unusual. As discussed in [11], RIA in $1440 \mathrm{~nm}$ region can be related with a formation of conjugated systems that are stable and expected to cause permanent RIA. Nevertheless, this phenomena's origin should be additionally investigated.

The dynamics of the above mentioned effects is more clearly seen on the graphs of the RIA evolution versus time passed after the end of the irradiation (Fig. 7). The graphs are plotted for two wavelengths: $880 \mathrm{~nm}$ representing the left side of the spectrum, and the RIA peak wavelength of $1440 \mathrm{~nm}$. The transmission recovery with time inherent for the left side of the spectrum is clearly seen in Fig. 7a. Moreover, a strong decrease of the RIA is observed during approximately five hours after irradiation. At $1440 \mathrm{~nm}$ (Fig. 7b), strong rise of the RIA also during approximately five hours after irradiation is clearly seen for the dose of $17.5 \mathrm{kGy}$; then the RIA rises more slowly tending to be saturated. The lower dose of $5 \mathrm{kGy}$ does not demonstrate rapid RIA growth in the beginning part of the graph and rises more smoothly, also tending to be saturated. It should be mentioned that during 2-2.5 hours after irradiation, the RIA at $1440 \mathrm{~nm}$ (Fig. 7b) demonstrates lower values in the case of $17.5 \mathrm{kGy}$ dose than the $5 \mathrm{kGy}$ case. We also can notice that the strong growth of the RIA occurs after but not during irradiation. These effects are not clear and future RIA investigation not only after, but also during irradiation process would clarify this behavior.

\section{CONCLUSIONS}

In this work, we experimentally investigated long-term postirradiation RIA variations of the CYTOP fiber. RIA measurements performed five months after irradiation demonstrated spectra qualitatively similar to those measured during and after irradiation and reported in previous works [7,11]. The main features of the long-term RIA are (1) significant decrease in time in visible range (however residual RIA is still present in the fiber even few months after irradiation), and (2) post-irradiation rise and saturation at wavelengths $>1300 \mathrm{~nm}$ (this RIA can be considered as permanent). The first feature should be taken into account when considering CYTOP fiber dosimetry applications based on the RIA: the RIA values strongly depend on time passed after irradiation. The second feature suggests to consider alternative wavelength range for dosimetry application where the RIA stabilization takes time, but the RIA values become permanent.
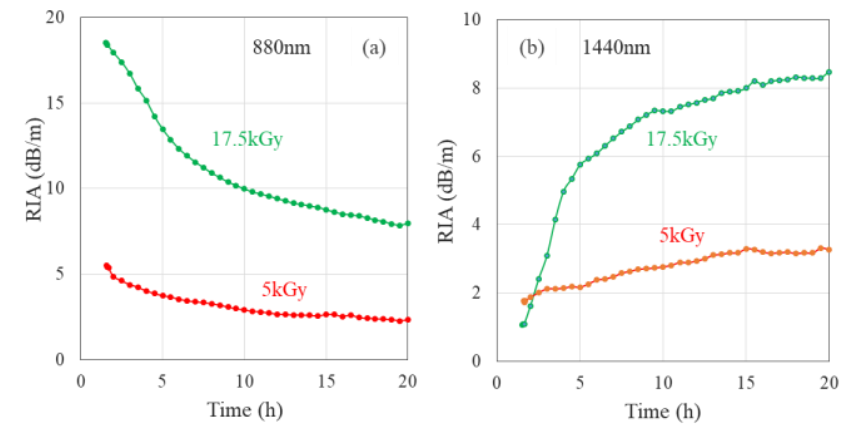

Fig. 7. RIA time evolution for $5 \mathrm{kGy}$ and $17.5 \mathrm{kGy}$ irradiation doses at $880 \mathrm{~nm}$ (a) and $1440 \mathrm{~nm}$ (b) wavelengths. The time is counted from the end of the irradiation.
Considering data transmission, the wavelength range 1000$1300 \mathrm{~nm}$ has minimum attenuation in long-term taking into account both RIA and pristine fiber losses. Partial transmission recovery with time in $850 \mathrm{~nm}$ window gives the opportunity to use this transparency window as well, if the method of further RIA reduction could be found.

\section{REFERENCES}

[1] K. Peters, "Polymer optical fiber sensors-a review," Smart Mater. Struct., vol. 20, no. 1, 2011, Art. no. 013002.

[2] L. Bilro, N. Alberto, J. L. Pinto, and R. Nogueira, "Optical sensors based on plastic fibers," Sensors (Switzerland), vol. 12, no. 9, pp. 12184-12207, 2012.

[3] Y. Koike and M. Asai, "The future of plastic optical fiber," NPG Asia Mater., vol. 1, no. 1, pp. 22-28, 2009.

[4] A. Theodosiou and K. Kalli, "Recent trends and advances of fibre Bragg grating sensors in CYTOP polymer optical fibres," Opt. Fiber Technol., vol. 54, 2020, Art. no. 102079 .

[5] Y. Mizuno and K. Nakamura, "Potential of Brillouin scattering in polymer optical fiber for strain-insensitive high-accuracy temperature sensing," Opt. Lett., vol. 35, no. 23, pp. 3985-3987, 2010.

[6] I. Chapalo, A. Theodosiou, K. Kalli, and O. Kotov, "Multimode Fiber Interferometer Based on Graded-Index Polymer CYTOP Fiber," J. Lightw. Technol., vol. 38, no. 6, pp. 1439-1445, 2020.

[7] P. Stajanca and K. Krebber, "Radiation-induced attenuation of perfluorinated polymer optical fibers for radiation monitoring," Sensors (Switzerland), vol. 17, no. 9, 2017, Art. no. 1959.

[8] C. Broadway, D. Kinet, A. Theodosiou, K. Kalli, A. Gusarov, C. Caucheteur, and P. Mégret, "CYTOP fibre Bragg grating sensors for harsh radiation environments," Sensors (Switzerland), vol. 19, no. 13, 2019, Art. no. 2853.

[9] P. Stajanca and K. Krebber, "Towards on-line radiation monitoring with perfluorinated polymer optical fibers," Proc. SPIE, vol. 10323, 2017, Art. no. 1032310 .

[10] P. Stajanca, L. Mihai, D. Sporea, D. Negut, H. Sturm, M. Schukar, and K. Krebber, "Impacts of gamma irradiation on Cytop plastic optical fibres," in The $25^{\text {th }}$ International Conference on Plastic Optical Fibers (POF2016), Birmingham, UK, 2016, pp. 114-117.

[11] P. Stajanca, L. Mihai, D. Sporea, D. Negut, H. Sturm, M. Schukar, and K. Krebber, "Effects of gamma radiation on perfluorinated polymer optical fibers," Opt. Mater., vol. 58, pp. 226-233, 2016. 